\title{
Pigment epithelium-derived factor short peptides facilitate full-thickness cutaneous wound healing by promoting epithelial basal cell and hair follicle stem cell proliferation
}

\author{
SHAWN H. TSAI ${ }^{1,2^{*}}$, LI-PENTSAO ${ }^{3 *}$, SHIH-HSIN CHANG ${ }^{4,5}$, \\ TSUNG-CHUAN HO ${ }^{3}$, KWANG-YI TUNG ${ }^{4}$, AI-CHING WU ${ }^{1}$ and YEOU-PING TSAO ${ }^{1}$ \\ ${ }^{1}$ Department of Ophthalmology, Mackay Memorial Hospital, Taipei 10449; ${ }^{2}$ Department of Optometry, \\ Chung Shan Medical University, Taichung 40201; Departments of ${ }^{3}$ Medical Research and ${ }^{4}$ Plastic Surgery, \\ Mackay Memorial Hospital, Taipei 10449; ${ }^{5}$ Department of Biomedical Engineering, \\ National Yang Ming University, Taipei 11221, Taiwan, R.O.C.
}

Received August 2, 2015; Accepted December 12, 2016

DOI: $10.3892 / \mathrm{etm} .2017 .5134$

\begin{abstract}
A previous study by our group showed that a 44-amino-acid fragment of pigment epithelium-derived factor (PEDF) facilitated corneal epithelial wound healing. In the present study this fragment was shortened to obtain peptides of 18,20 and 29 amino acids in length, and their promoting effects on the healing of full-thickness skin wounds were assessed. Peptides were delivered periodically by topical application to punch wounds of mice. The wound healing speed was evaluated by measuring the reduction of wound areas at 4 and 7 days after injury. Histological analysis with Masson's trichrome staining was used to confirm epithelialization and dermal collagen deposition. Proliferation of epithelial basal cells was documented by 5-bromo-2'-deoxyuridine incorporation. Hair follicle stem cells were identified by immunostaining for leucine-rich repeat-containing $\mathrm{G}$ protein-coupled receptor 6 . The results indicated that the 20- and 29-amino-acid short peptides significantly reduced the time required for wound healing compared to the vehicle. Histological analysis confirmed faster epithelial cell coverage of open wounds. Treatment with the PEDF peptide fragments also contributed to granulation, tissue formation by increasing the fibroblast population and enhancing collagen deposition in the dermis. Wounds treated with PEDF peptide fragments contained more basal cells proliferated in the epithelium. Moreover, hair follicle stem cells were also stimulated to proliferate by peptide
\end{abstract}

Correspondence to: Dr Yeou-Ping Tsao, Department of Ophthalmology, Mackay Memorial Hospital, 92 Sector 2 Zhongshan North Road, Taipei 10449, Taiwan, R.O.C.

E-mail: yptsao@yahoo.com

*Contributed equally

Key words: pigment epithelium-derived factor, peptide, hair follicle stem cell, wound healing, epithelialization exposure. In conclusion, the present study reported the identification of two short peptides that can enhance the healing of full-thickness skin wounds following topical application. The underlying mechanisms may involve activation of basal cell proliferation and mobilization of hair follicle stem cells.

\section{Introduction}

Commonly observed chronic cutaneous venous ulcers, diabetic foot ulcers and pressure ulcers heal with difficulty and cause significant morbidity for patients due to a delayed healing process and secondary complications. These skin injuries pose a huge financial burden upon national health systems and constitute one of the important issues that require immediate attention (1). At present, the clinical understanding is insufficient and the development of novel treatments to accelerate wound healing is required (2).

In response to full-thickness skin lesions, a complex, multi-phase process is activated, involving inflammation, proliferation and maturation. This eventually results in the formation of scar tissue and leads to the proper restoration of skin integrity and barrier function (3). In the initial stage of inflammation, inflammatory cells participate in hemostasis and the clearing of wound debris. This inflammatory process is primarily meditated by factors, including platelet-derived growth factor or tumor necrosis factor $\alpha$, released from mast cells and platelets, which initiate angiogenesis and preliminary repair within $48 \mathrm{~h}$ of injury (4-6). During the subsequent proliferation stage, granulation tissue (GT) provides the growth of a new provisional wound matrix and promotes angiogenesis. Proliferation of epithelial progenitor cells helps achieve re-epithelialization of the wound bed and re-establishes the outer protective barrier (4). Together, keratinocytes and fibroblasts stimulate each other to release growth factors, which in turn stimulate cellular growth at the wound site, leading to proper wound closure $(3,7)$. Finally, the maturation stage involves wound contraction to decrease the size of an open wound and synthesis of collagen by fibroblasts to re-build dermal tissue. However, this process requires the proper 
alignment of the collagen bundles and fibroblasts, which is also meditated by GT (8). Full completion of wound healing may take months or years, requiring dermal tissue remodeling and building of tensile strength, and in most cases, full recovery is not achieved and permanent scars remain at wound sites.

A previous investigation indicated the participation of hair follicle stem cells (HFSCs) in wound healing. Under normal conditions, they are not involved in the regular turnover of cutaneous epithelium (9). Upon wounding, HFSCs residing in the hair follicles migrate to the epidermis to aid in re-epithelialization (10). In this regard, the role of HFSCs in skin re-epithelialization suggests their potential application in an approach of exogenous stimulation of wound healing.

Prompt re-epithelialization and the closure of open wounds are crucial to prevent excessive inflammation, scar formation and opportunistic infection. A therapeutic approach is to facilitate the proliferation of epithelial progenitor cells by growth factors. A good example of such a growth factor is platelet-derived growth factor (PDGF). Previously, a topical gel formulation containing PDGF has been shown to be capable of stimulating epithelial cell proliferation and accelerating wound healing (11). Such growth factor therapy has been used in the clinic to treat patients with diabetes-associated skin ulcers with delayed healing (12).

Pigment epithelium-derived factor (PEDF) is a protein containing peptide domains responsible for neurotrophic and anti-angiogenesis functions. PEDF is produced by keratinocytes in the wound area and changes in PEDF levels have been correlated with skin ageing and pathological injuries (13). Therefore, it has been postulated that PEDF may have an important role in early wound repair and is associated with tissue homeostasis. A previous study by our group identified that a 44-amino-acid fragment of PEDF, which is known to have a neurotrophic function, stimulates progenitor cell proliferation and corneal epithelial wound healing (14). The study indicated that this PEDF fragment stimulated progenitor cell proliferation within the limbal stem cell niche and in the basal layer of healing epithelium. A recent study by our group found that the 44-amino-acid fragment of PEDF also promoted muscle regeneration by stimulating the proliferation of muscle stem cells and satellite cells (15). These finding suggested that PEDF fragments may also be able to facilitate skin wound healing. In the present study, the previously used 44-amino-acid peptide was shortened to 18-, 20- and 29-amino-acid peptides and their effect in promoting the healing of full-thickness skin wounds in mice and the proliferation of human follicle stem cells was assessed. It was revealed that the peptide fragments not only promoted re-epithelization of skin wounds but also increased the local accumulation of dermal collagen.

\section{Materials and methods}

Materials. HEPES-buffered Dulbecco's modified Eagle's medium (DMEM), Ham's/F12 medium, trypsin-EDTA, fetal bovine serum (FBS), bovine pituitary extract, antibiotic-antimycotic solutions and trypsin were from Invitrogen (Thermo Fisher Scientific, Inc., Waltham, MA, USA). Masson's trichrome stain, dimethyl sulfoxide (DMSO), mitomycin C (MMC), 5-bromo-2'-deoxyuridine (BrdU), insulin-transferrin-sodium selenite (ITSE) media supplement and Hoechst 33258 dye were all from Sigma-Aldrich (Merck-Millipore, Darmstadt, Germany). Collagenase A and dispase II were obtained from Roche (Indianapolis, IN, USA). Antibodies against leucine-rich repeat-containing $\mathrm{G}$ protein-coupled receptor 6 (Lgr6; cat. no. sc-99123; Santa Cruz Biotechnology, Dallas, TX, USA) and BrdU (cat. no. GTX42641; GeneTex, San Antonio, TX, USA) were used as primary antibodies. Rhodamine-conjugated donkey anti-mouse immunoglobulin (Ig)G (cat. no. AP192R; EMD Millipore, Billerica, MA, USA) and fluorescein isothiocyanate (FITC)-conjugated donkey anti-rabbit IgG were used as secondary antibodies (cat. no. 406403; BioLegend, San Diego, CA, USA). Human plasma was obtained from two of the authors of the current study (YPT and TCH). PEDF was purified from this human plasma via collagen I-sepharose resin, as described previously (16) and preserved in a buffer composed of $20 \mathrm{mM}$ sodium phosphate, $\mathrm{pH}$ 6.4, 0.2 $\mathrm{M}$ sodium chloride and $1 \mathrm{mM}$ dithiothreitol. Short synthetic PEDF peptides, including the 29-mer (residues Ser93-Thr121), 20-mer (Ser93-Leu112) and 18-mer (Glu97-Ser114) were synthesized, modified by acetylation at the $\mathrm{NH}_{2}$ termini and amidation at the $\mathrm{COOH}$ termini for stability, and characterized by mass spectrometry ( $>90 \%$ purity), to order by GenScript (Piscataway, NJ, USA).

Analysis of skin wound healing. Experimental procedures were approved by the Mackay Memorial Hospital Review Board (Taipei, Taiwan) and performed according to national animal welfare regulations (Council of Agriculture, R.O.C). To perform full-thickness skin excision, 64 8-10 week-old female C57BL/6 mice $(20 \pm 2 \mathrm{~g})$ were purchased from BioLASCO Taiwan Co., Ltd. (Taipei, Taiwan) and housed in a $25^{\circ} \mathrm{C}$ temperature-controlled room with a 12-h light/dark cycle. Mice had ad libitum access to water and pelleted standard laboratory chow (BioLASCO Taiwan Co., Ltd.). Mice were anesthetized by an intraperitoneal injection of a mixture of zoletil (6 mg/kg; Virbac Laboratories, Carros Cedex, France) and Rompun ${ }^{\circledR}$ (3 mg/kg; Rompun Bayer Korea Ltd., Seoul, Korea). Two full-thickness skin excisional wounds $4 \mathrm{~mm}$ in diameter were made on each side of the dorsal midline using a Sklar Tru-Punch disposable biopsy punch (Sklar, West Chester, PA, USA). PEDF-derived short peptide was reconstituted in DMSO as stock $(5 \mathrm{mM})$ and mixed with TOBREX eye ointment containing $0.3 \%$ tobramycin and $0.5 \%$ chlorobutanol (Alcon, Fort Worth, TX, USA) to achieve a PEDF fragment concentration of $50 \mu \mathrm{M}$. In each treatment group, the skin wound was treated with $25 \mu \mathrm{l}$ of this ointment topically (containing $0.25 \mu \mathrm{DMSO}$ as vehicle) once daily after scraping of the skin. In the vehicle control group, the ointment only contained an equal volume of DMSO. Mice were housed individually during the healing period.

The dimensions of the wound areas were quantified from images captured using a computer-assisted image analyzer (Adobe Photoshop CS3 10.0; Adobe Systems, San Jose, CA, USA) and the percentage of residual epithelial defect at each time-point vs. the initial wound area was calculated.

Prior to histological analysis, mice $(n=8$ per time point and 2 wounds per mouse) were euthanized by $\mathrm{CO}_{2}$ (flow rate: $2 \mathrm{l} / \mathrm{min}$; final $\mathrm{CO}_{2}$ concentration $>70 \%$; euthanasia confirmed by cardiac and respiratory arrest). The complete wound covering $2 \mathrm{~mm}$ of the epithelial margins was isolated, 
bisected, fixed overnight in $4 \%$ paraformaldehyde in PBS and embedded in paraffin. Sections $(5 \mu \mathrm{m})$ from the middle of the wound were stained using the Masson's trichrome procedure as described by the manufacturer, in which the epithelium was stained red and connective tissue was stained blue. Images were captured using a Nikon Eclipse 80i microscope (Nikon Corporation, Tokyo, Japan) equipped with a Leica DC 500 camera (Leica Microsystems, Wetzlar, Germany).

To measure cell proliferation, BrdU (Sigma-Aldrich; Merck Millipore) was used after reconstitution in DMSO at $(80 \mathrm{mM})$. Of this BrdU stock, $10 \mu \mathrm{l}$ was mixed with $90 \mu \mathrm{l}$ PBS and injected intraperitoneally into the mice at $6 \mathrm{~h}$ prior to euthanasia with $\mathrm{CO}_{2}$. . DNA synthesis in proliferating cells was assessed by measurement of BrdU incorporation.

Cultivation of human HFSCs. Human hair follicles were isolated from the hairy scalp skin of healthy donors. Written informed consent was obtained from all donors Human tissue was handled according to the tenets of the Declaration of Helsinki. The protocol was approved by the Institutional Review Board of the Mackay Memorial Hospital (Taipei, Taiwan). The follicles were dissected with tweezers under microscopy and the majority of adipose and connective tissue was removed. The bulb region of the hair follicle under the sebaceous gland was cut and bulb fragments were transferred into a $35-\mathrm{mm}$ dish containing $1 \mathrm{ml}$ collagenase A $(1 \mathrm{mg} / \mathrm{ml})$, followed by incubation for $1.5 \mathrm{~h}$ at $37^{\circ} \mathrm{C}$ to digest the collagen capsule. Subsequently, the bulb fragments were transferred into a fresh cell culture dish containing dispase II (2.4 U/ml) $/ 0.05 \%$ trypsin and incubated for $1.5 \mathrm{~h}$ at $37^{\circ} \mathrm{C}$ to obtain a single-cell suspension.

After mechanical dissection and enzymatic digestion, the suspended cells isolated from five bulb fragments were seeded into one well of a 12-well culture plate and incubated with a basal medium supplement containing four parts calcium-free DMEM and one part Ham's F12 medium (final calcium concentration, $0.25 \mathrm{mM}$ ). The basal medium was further supplemented with $10 \mathrm{ng} / \mathrm{ml}$ human epidermal growth factor (cat. no. AF-100-15; PeproTech EC Ltd., Rocky Hill, NJ), 500 mg/l L-glutamine (Gibco; Thermo Fisher Scientific, Inc.), $0.2 \%$ bovine pituitary extract, $0.18 \mathrm{~g} / \mathrm{ml}$ hydrocortisone (Gibco; Thermo Fisher Scientific, Inc.), 1\% ITSE as well as antibiotic-antimycotic solution and $10 \%$ FBS to support cell growth. The cultivation method was based on that of a previous study (17). The medium was changed every 2-3 days. The bulb cells were co-cultured with MMC ( $4 \mu \mathrm{g} / \mathrm{ml} ; 2 \mathrm{~h})$-treated NIH/3T3 fibroblast feeder cells (CRL-1658 ${ }^{\mathrm{TM}}$; ATCC, Manassas, VA, USA). At the third passage, near-confluent cells were harvested with $0.25 \%$ trypsin for $5 \mathrm{~min}$ at $37^{\circ} \mathrm{C}$ for use in the BrdU labeling assay.

BrdU labeling. Approximately $1 \times 10^{5}$ human bulb cells were transferred to a fresh culture dish and allowed to grow for 2 days. Subsequently, cells were cultured in a basal medium (untreated; UT) or basal medium containing either $4.5 \mathrm{nM}$ of the 44-amino-acid fragment of PEDF or $50 \mathrm{nM}$ of the 22-amino-acid fragment of PEDF for $24 \mathrm{~h}$. BrdU (final concentration, $10 \mu \mathrm{M}$ ) was added to the culture for $2 \mathrm{~h}$. Following fixation with $4 \%$ paraformaldehyde at room temperature for
$2 \mathrm{~h}$, cells were exposed to cold methanol at $4^{\circ} \mathrm{C}$ for 2 min and then treated with $1 \mathrm{~N} \mathrm{HCl}$ at $\mathrm{RT}$ for $1 \mathrm{~h}$ prior to immunofluorescence microscopy.

Immunofluorescence. Deparaffinized tissue sections or $4 \%$ paraformaldehyde-fixed human bulb cells were blocked with 10\% goat serum (Gibco; Thermo Fisher Scientific, Inc.) and 5\% bovine serum albumin (Gibco; Thermo Fisher Scientific, Inc.) in PBS containing 0.1\% Tween-20 for $1 \mathrm{~h}$. Staining was performed using primary antibodies against Lgr6 (1:100 dilution) or BrdU (1:100 dilution) at $37^{\circ} \mathrm{C}$ for $2 \mathrm{~h}$, followed by incubation with the appropriate rhodamine- or FITC-conjugated donkey IgG (1:500 dilution) for $1 \mathrm{~h}$ at room temperature. Images were captured using a epifluorescence microscope (Zeiss Axioplan 2 imaging; Zeiss, Oberkochen, Germany) equipped with a charge-coupled device camera (Zeiss AxioCam HRm, Zeiss) and quantification was performed using Axiovert software (Zeiss AxioVision Release 4.8.2, Zeiss).

Statistical analysis. Data were analyzed using Microsoft Excel 2000 version 9 (Microsoft Corporation, Redmond, WA, USA) and expressed as the mean \pm standard error of the mean. One-way analysis of variance was used for statistical comparisons. $\mathrm{P}<0.05$ was considered to indicate a statistically significant difference.

\section{Results}

PEDF-derived short peptides accelerate skin wound healing. To investigate the effects of the PEDF-derived short peptides on skin wound healing, 4-mm punch wounds were made on the dorsal side of C57BL/6 mice and subsequently treated with ointment containing 29-mer, 20-mer, 18-mer or DMSO vehicle. As shown in Fig. 1, 29- and 20-mer-treated groups showed significantly enhanced wound healing at day 4 post-injury compared to the vehicle group $(46.3 \pm 3.7$ and $50.1 \pm 3.8$ vs. $61.5 \pm 3.2 \%$ epidermal defect remaining). At day 7 post-injury, skin wounds receiving 29- and 20-mer peptides showed almost complete healing and substantially less scar tissue developed, compared to vehicle-treated wounds $(18.2 \pm 4.3$ and $20.0 \pm 4.9$ vs. $44.5 \pm 5.3 \%$ epidermal defect remaining). The results of the current study indicated that control peptide (18-mer) treatment was not able to promote wound healing. The results indicated that the 29- and 20-mer benefit skin re-epithelialization.

Histology of partially healed wounds treated with PEDF-derived short peptides. To confirm the efficacy of PEDF-derived short peptides in skin wound repair, sections of wound tissue from day 4 after wounding were stained using Masson's trichrome. As shown in Fig. 2A, 29-mer-treated skin wounds showed improved wound healing than those in the vehicle-treated group. The residual epithelial defects in the

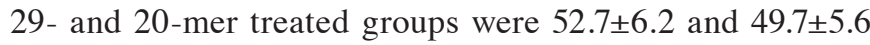
compared to $63.8 \pm 5.8 \%$ for the vehicle group (Fig. 2B).

In addition, PEDF 29-mer treatment increased the thickness of the hyperproliferative epithelium (HE) and GT at the wound edge (Fig. 2C). Quantification of the HE tissue area revealed that total area growth in the 29- and 20-mer-treated 
A

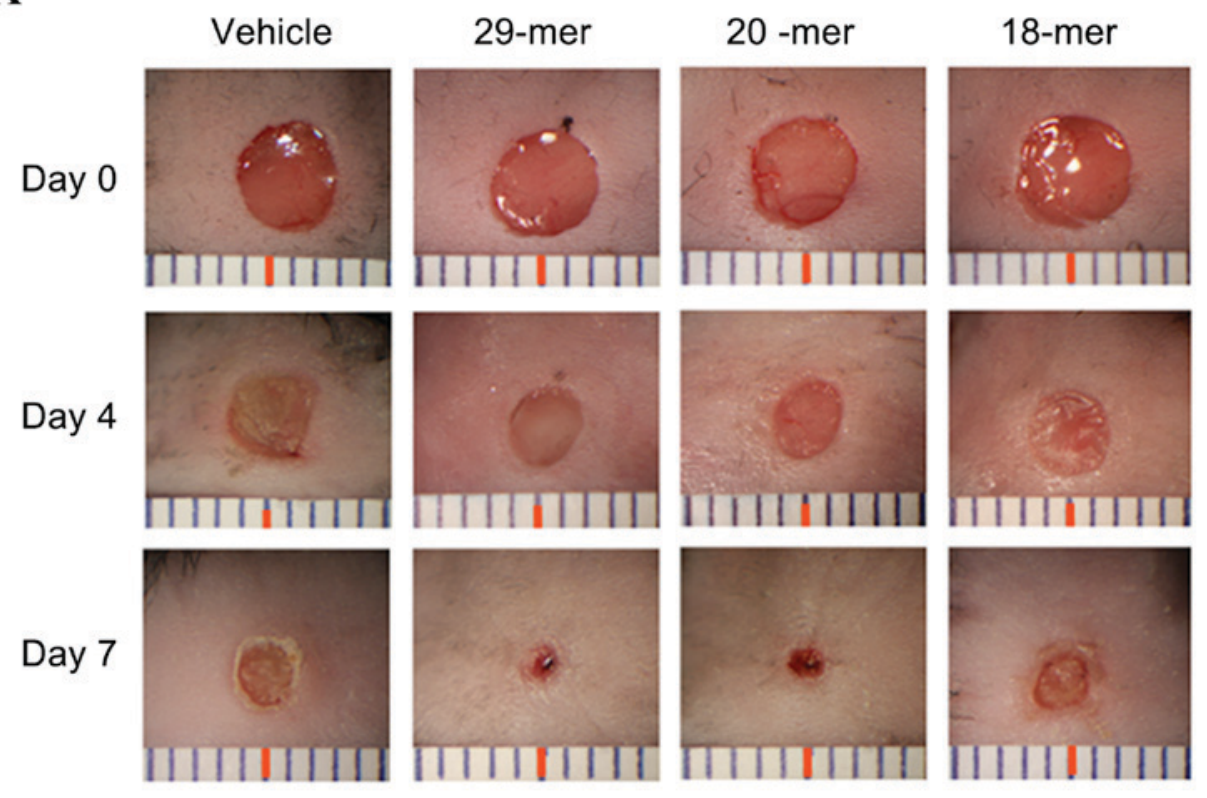

B

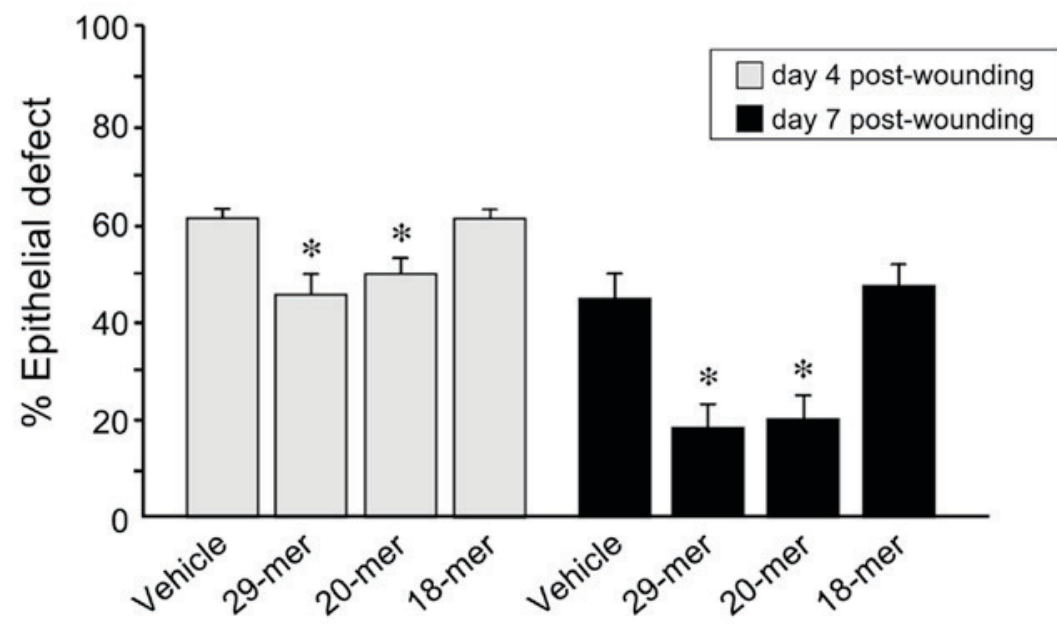

Figure 1. Short peptides derived from pigment epithelium-derived factor promote skin wound healing. (A) 4-mm punch wounds were made on the dorsal side of C57BL/6 mice. Skin ointment was applied once per day. Images of the wounded skin and a ruler (scale, $10 \mathrm{~mm}$ ) were captured. (B) The residual epithelial defect $(\%)$ is presented as a percentage of the original wound size. Values are expressed as the mean \pm standard error. Four independent experiments were performed with four mice per group. Wound healing was significantly improved by the 20 - and 29 -mer at 4 and 7 days. "P<0.05 vs. vehicle-treated wounds.

groups was $1.41 \pm 0.25$ - and $1.32 \pm 0.21$-fold higher, respectively, than that in the vehicle-treated group (Fig. 2D). Furthermore, quantification of the area of GT indicated that the 29- and 20 -mer-treated groups had a $1.45 \pm 0.23$ and $1.37 \pm 0.17$-fold higher area recovery than the vehicle group (Fig. 2E). Skin sections from day 7 after trauma were stained using Masson's trichrome to measure wound closure. The 29- and 20-mer-treated wounds showed better re-epithelialization evidenced by the smaller residual epithelial defects compared with those of vehicle ointment-treated wounds $(17.9 \pm 3.3$ and 17.2 \pm 7.3 vs. $43.5 \pm 6.5 \%$; Fig. 3). Collectively, the 29 -mer and the 20-mer increased the growth of HE and GT, resulting in the acceleration of wound closure.

PEDF-derived short peptides accelerate skin wound healing by promoting proliferation of the basal cells of the HE. To investigate whether skin resurfacing accelerated by treatment with PEDF-derived short peptides involves epithelial basal cell replication, wounds were treated with PEDF-derived peptide ointments for 4 days and the mice were then intraperitoneally injected with BrdU and sacrification at $6 \mathrm{~h}$ thereafter. Immunohistochemical analysis of skin specimens using the anti-BrdU antibody showed that the BrdU-positive cells were are mostly distributed in the basal layer of HE tissue and the bulb region of hair follicles (Fig. 4A). The numbers of BrdU-positive cells were significantly increased in 29- and 20-mer-treated wounds compared to those in vehicle-treated wounds $(47.9 \pm 5.0$ and $53.1 \pm 6.5$ vs. $30.8 \pm 8.1 \%$; Fig. 4B). Noticeably, HE tissues in wounds treated with PEDF 29-mer peptides were thicker than vehicle-treated group (Fig. 4A), which would be expected due to the increase of actively proliferating basal cells. These evidences indicated that PEDF peptides stimulate epithelial basal cell proliferation in healing wounds. 
A
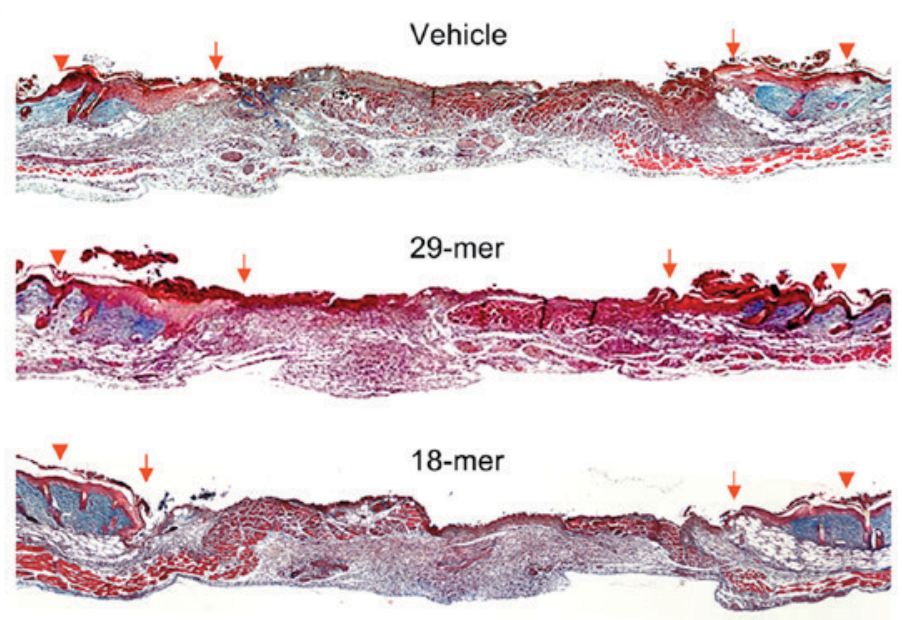

C
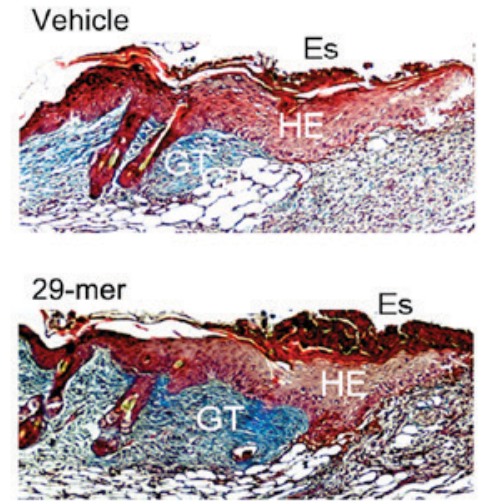

D

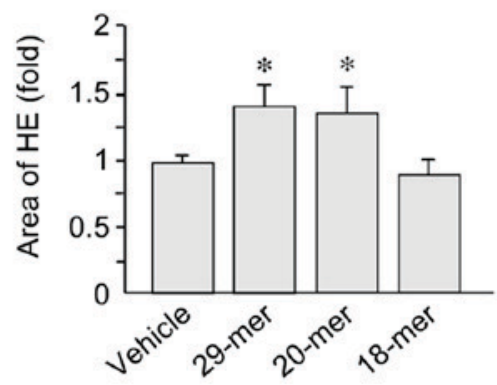

B

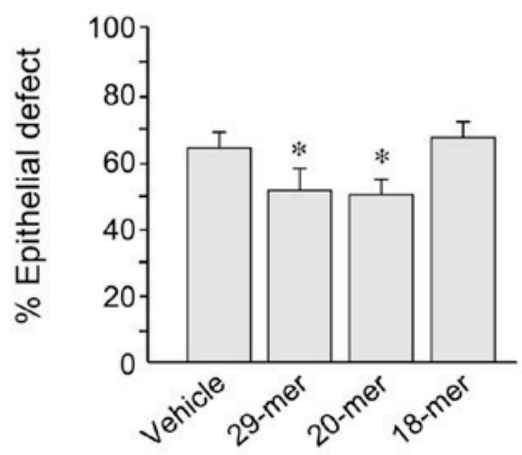

Day 4 post-wounding

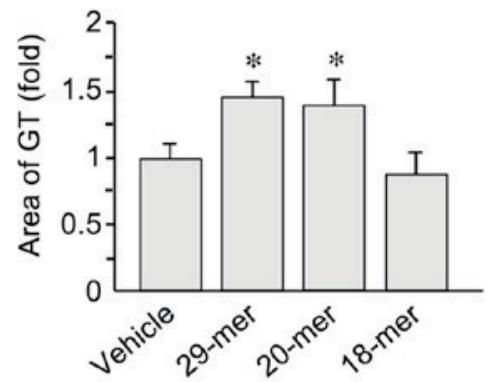

Figure 2. Quantification of wound healing at day 4. (A) Representative images of skin specimens stained by Masson trichrome (original magnification, x200). The 29-mer -treated wounds showed improved wound healing. Arrowheads and arrows denote initial wound margins and the foremost tips of the HE (red color), respectively. Representative results from four separate experiments are shown. (B) The residual epithelial defect (\%) was determined in control ointment- and peptide-treated mice; only sections of the middle of the wounds were used for quantification. (C) Representative images of wound edges. 29-mer-treated wounds showed thicker HE and GT (blue color). The Es stained deep red. Representative results from four separate experiments are shown. (D and E) Quantification of the areas of HE and GT. For each group, 10-16 wounds (2 wounds/mouse) were assessed. Values are expressed as the mean \pm standard error. "P<0.02 vs. control wounds. HE, hyperproliferative epithelium; GT, granulation tissue; ES, eschar.

PEDF-derived peptide promotes Lgr6-positive cell proliferation during skin wound healing. Lgr6-positive HFSCs are important precursor cells for the repair of epithelium during skin wound healing (18). To investigate the participation of HFSCs in wound healing, a Lgr6 and BrdU double immunostaining experiment was performed and the involvement of proliferating Lgr6-positive HFSCs was determined. As shown in Fig. 5A, immunostaining confirmed that Lgr6-positive cells (green) were distributed in HE tissue after skin injury. Moreover, dual immunostaining analysis of the skin specimens from wounds treated with 20-mer ointment for 4 days showed that the BrdU/Lgr6-double positive cells were markedly increased in HE tissue, compared to those in vehicle-treated wounds ( $46.7 \pm 5.8$ vs. $26.0 \pm 3.6 \%$; Fig. $5 \mathrm{~B}$ ). This indicates the participation of HFSCs in wound healing following stimulation by the 20-mer fragment of PEDF.

PEDF and 29-mer promote HFSC proliferation in vitro. To determine the influence of the 44-mer fragment of PEDF and its short derivatives on HFSC proliferation, human hair follicles cultured and treated with PEDF were stained for HFSC markers. As shown in Fig. 6A, BrdU pulse-labeling (2 h; red color) was performed and the HFSC phenotype was confirmed by immunostaining of Lgr6 marker (green color). Human HFSCs cultured in medium containing PEDF or 20-mer exhibited a significantly higher proliferative rate than the untreated group cultivated in control medium $(73.8 \pm 6.0$ and $79.5 \pm 2.6$, respectively, vs. $31.9 \pm 5.6 \%$; Fig. $6 \mathrm{~B})$. Collectively, these results indicated that PEDF or its derived 20-mer enhanced HFSC proliferation in vitro.

\section{Discussion}

In the present study, by exposing cutaneous punch wounds to PEDF peptide, improved wound healing and HFSC expansion was demonstrated in vivo. This was observed at 4 and 7 days after wounding. Furthermore, histological analysis indicated faster wound coverage by keratinocytes and accumulation of GT under PEDF peptide fragment treatment. To the best of our knowledge, the present study was the first to describe the healing of full-thickness skin wounds following treatment with the PEDF peptide derivatives.

In the present study active proliferation of epithelium basal cells under PEDF peptide treatment was observed, particularly 
A
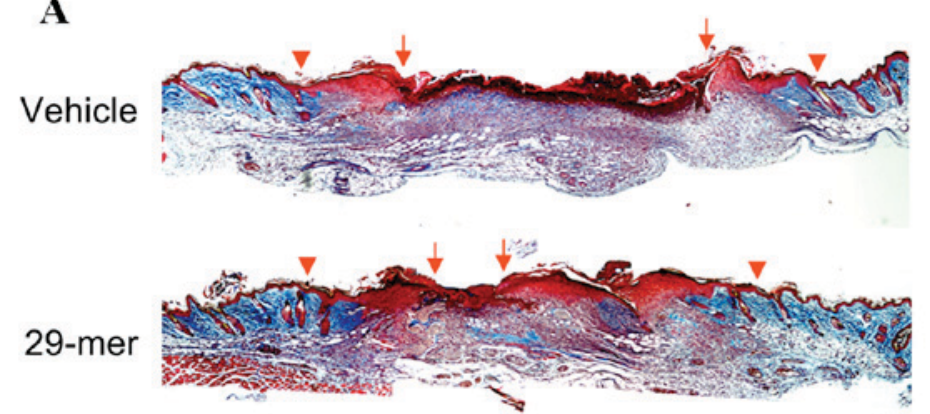

20-mer

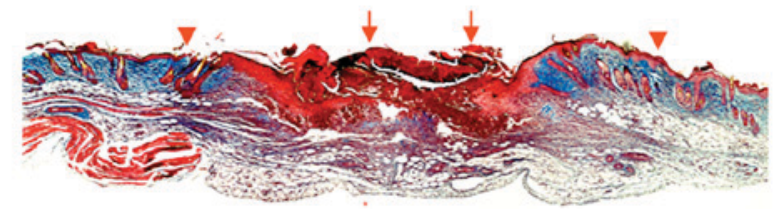

18-mer

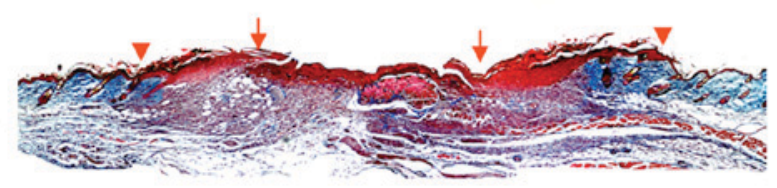

B

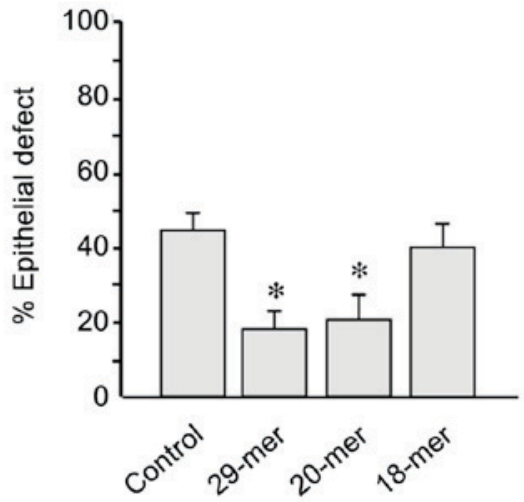

Day 7 post-wounding

Figure 3. Quantification of wound healing at day 7. (A) Representative images of skin specimens stained by Masson trichrome (original magnification, $\mathrm{x} 200$ ). The 29- and 20-mer-treated wounds showed better healing. Arrowheads and arrows denote initial wound margins and the foremost tips of the hyperproliferative epithelium, respectively. Representative results from four separate experiments are shown. (B) The residual epithelial defects (\%) in vehicle- and short peptide-treated mice were determined; only sections of the middle of the wounds were used for quantification. For each group, $10-16$ wounds ( 2 wounds/mouse) were assessed. Values are expressed as the mean \pm standard error. ${ }^{*} \mathrm{P}<0.02$ vs. control wounds.

A

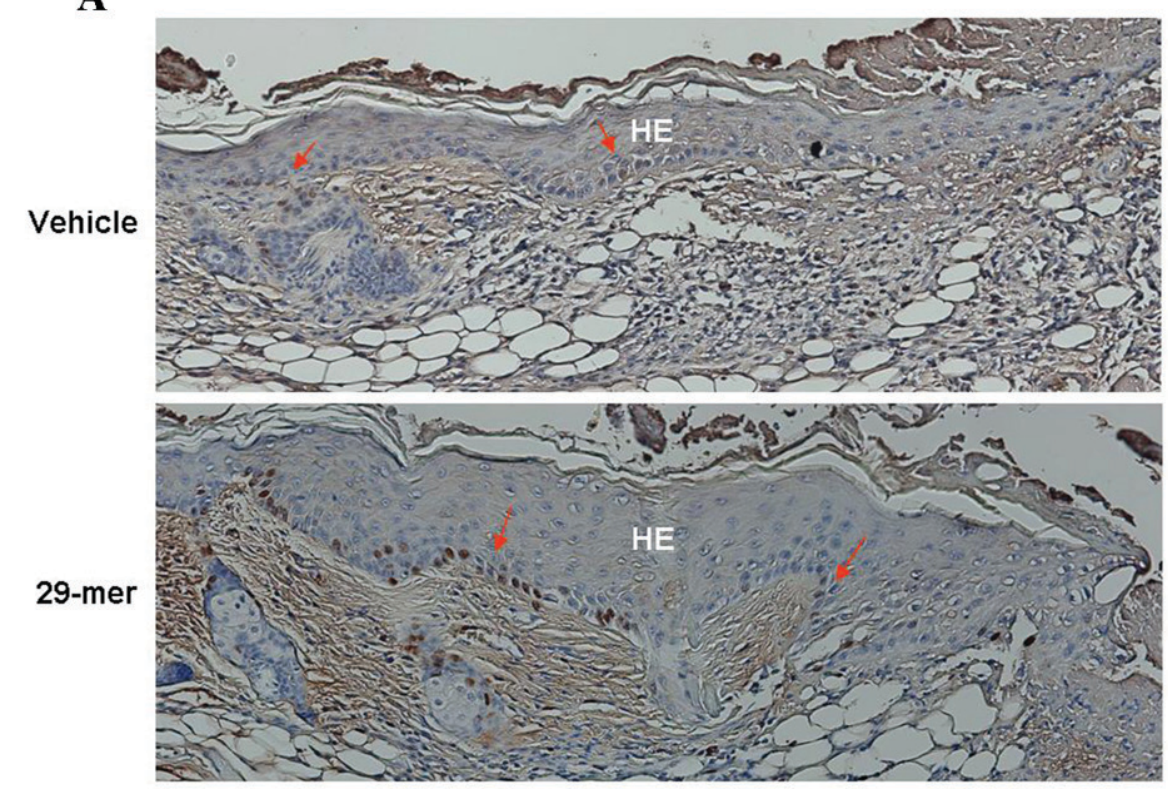

\section{B}

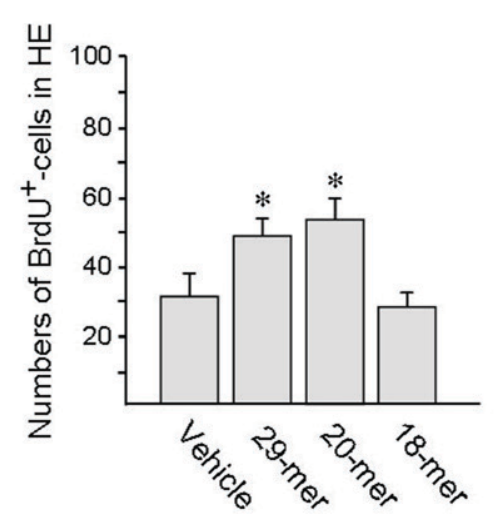

Day 4 post-wounding

Figure 4. Analysis of cell replication at day 4 post-skin wounding. (A) Specimens were histologically stained with BrdU to observe DNA replication (deep brown color) and counterstained with hematoxylin to observe nuclei. Representative images show cell replication mainly in the basal layer of HE as indicated by arrows (original magnification, x200). (B) Numbers of BrdU-positive cells in the basal layer of HE. The labeling index (\%) was calculated as the number of labeled cells divided by the total number of cells in the basal layer of HE. For each group, 10-16 wounds (2 wounds/mouse) were assessed. Values are expressed as the mean \pm standard error. ${ }^{~} \mathrm{P}<0.02$ vs. vehicle-treated group. HE, hyperproliferative epithelium; BrdU, 5-bromo-2'-deoxyuridine.

around the wound margin. One of the possible explanations for this is that PEDF peptide stimulates basal cell proliferation. Another possibility is that these basal cells were newly generated from a stem cell niche, such as the bulb of the hair follicle, and maintained their progenitor characteristic (Lgr6 marker) and superior proliferative potential. In PEDF peptide-assisted cornea wound healing, basal cells in the newly regenerated corneal epithelium have been previously reported to proliferate 
A

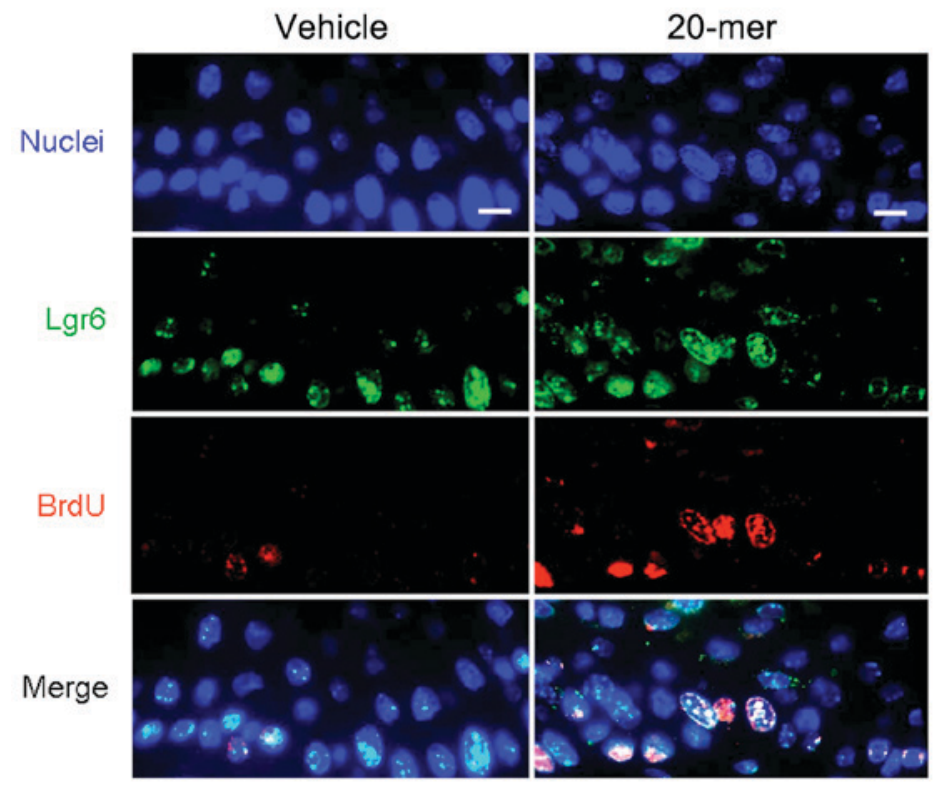

B

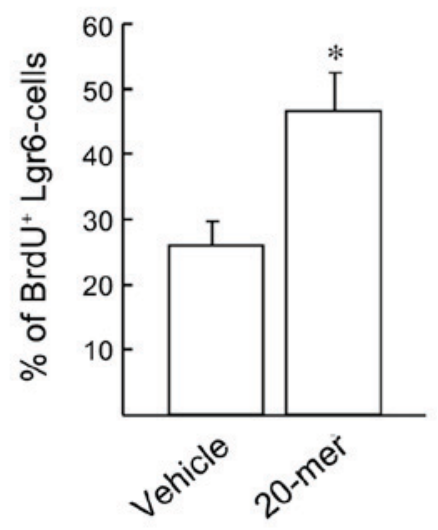

Figure 5. The 20-mer promotes Lgr6 cell proliferation after skin injury. (A) Immunofluorescence analysis of the levels of BrdU- and Lgr6-positive cells in the $\mathrm{HE}$ at $6 \mathrm{~h}$ post-wounding. Nuclei were visualized with Hoechst 33258 staining. Representative images show that 20 -mer treatment stimulated the expansion of Lgr6-positive cells for skin resurfacing. Scale bar=10 $\mu \mathrm{m}$. (B) The labeling index was calculated as the number of labeled cells divided by the total number of BrdU- and Lgr6-positive cells. Immunofluorescence was evaluated from 3 sections per mouse skin, and 6 mice in each group. "P $<0.01$ vs. vehicle-treated group. HE, hyperproliferative epithelium; BrdU, 5-bromo-2'-deoxyuridine; Lgr6, leucine-rich repeat-containing G protein-coupled receptor 6.

A

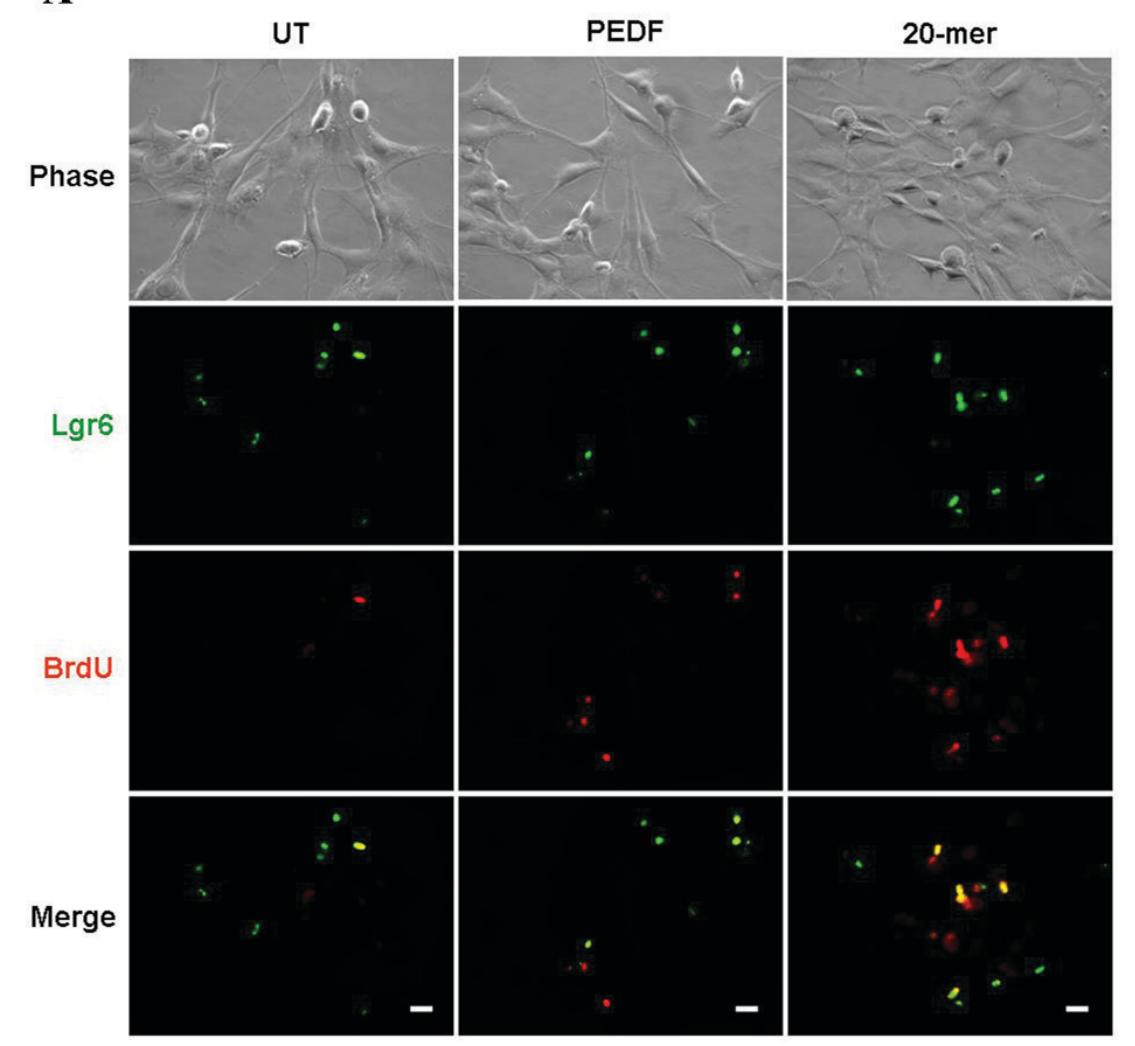

B

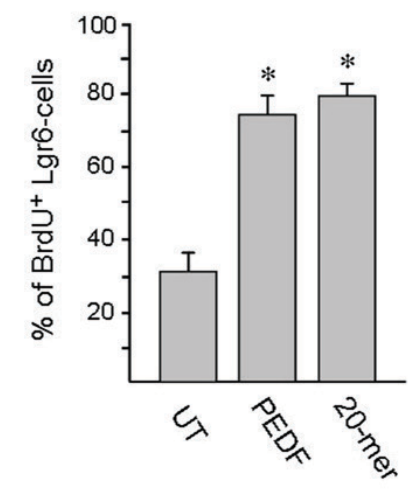

Figure 6. The 20- and 44-amino-acid fragments of PEDF enhance proliferation of HFSCs. Cell proliferation was determined by BrdU labeling for $2 \mathrm{~h}$. (A) Representative images of three independent experiments show phase contrast microscopy images as well as immunofluorescent staining of HFSCs for Lgr6 (green) and with BrdU (red), and merged signal (yellow). Original magnification, $\mathrm{x} 1,000$. Scale bar=10 $\mu \mathrm{m}$. (B) For each treatment, the percentage of BrdUand Lgr6-double positive cells (pale pink) per total Lgr6-positive cells was calculated from fifteen randomly selected fields. "P<0.001 vs. UT cells. PEDF, 44-amino-acid fragment of pigment epithelium-derived factor; BrdU, 5-bromo-2'-deoxyuridine; Lgr6, leucine-rich repeat-containing G protein-coupled receptor 6; UT, untreated; HFSCs, hair follicle stem cells. 
actively and maintain a high level of cornea progenitor cell marker $\Delta \mathrm{Np} 63 \alpha$ (14). The similar findings for skin epithelial basal cells and corneal basal cells suggest that skin epithelial basal cells may retain certain properties of progenitor cells under the influence of the PEDF peptide.

In addition to the stem cell proliferative effect, PEDF also has an anti-angiogenesis domain that lies within the 34-mer region (amino acid positions Asp44-Asn77) (19). However, angiogenesis is a function that participates in wound healing, via mast cell recruitment and subsequent initiation of GT and keratinocyte proliferation. By contrast, the anti-angiogenesis function is responsible for arresting neovascularization through reducing local vascular endothelial growth factor (VEGF) synthesis and suppression of VEGF receptor expression, which promotes endothelial cell apoptosis $(20,21)$. Therefore, the full-length PEDF protein containing the responsible 34-mer anti-angiogenesis domain may not be a good candidate for wound healing therapy. PEDF levels were found to be reduced locally after wound injuries, and exogenous PEDF was found to delay wound healing in diabetic animals (22). However, the synthetic 29- or 20-mer peptides did not include the 34-mer anti-angiogenesis domain of PEDF, and were successfully demonstrated to be accountable for the wound healing function of PEDF.

Previously, the documented association regarding PEDF and stem cell proliferation was limited to neural stem cells (NSCs). It has been reported that PEDF promotes the formation of neurospheres in vitro from NSCs derived from the hippocampus and neuroretina (23). Moreover, PEDF promotes NSC self-renewal by symmetrical division in the subventricular zone of the dentate gyrus in the hippocampus (24). A previous study by our group identified the expansion of limbal corneal epithelial stem cells by PEDF peptide (14). This indicated that the stem cell-promoting function of PEDF is not limited to the neuronal system. The observations made in the present study further expanded the scope of the function of PEDF to the proliferation of cutaneous epithelial stem cells and wound healing. PEDF appears to be functional in stem cell proliferation and expansion in multiple systems. Based on this knowledge, PEDF peptide may be useful for tissue regeneration therapy in various disease conditions.

Growth factors are important promoters of wound healing by controlling the growth, differentiation and metabolism of cells. Interleukin (IL)-1 released by keratinocytes induces fibroblasts to secrete important cytokines and growth factors, such as keratinocyte growth factor, fibroblast growth factor 7, IL-6 granulocyte-macrophage colony-stimulating factor and hepatocyte growth factor during wound repair (25-27). These factors in turn promote keratinocyte proliferation, in a paracrine-fashioned feedback loop, which is essential for proper healing of the wound site (3). PEDF was also found to impair skin wound healing in diabetic animals (22). The results of the present study may suggest that PEDF is a physiological reparative factor. However, it remains elusive whether PEDF is essential for wound healing, since the wound healing function of PEDF may be replaceable by other growth factors. However, effect of the topical application of short PEDF fragments shown in the present study indicated that they, similar to other reparative cytokines, promote wound healing.
HFSC is a cellular source for re-epithelialization. The present study provided evidence for HFSC proliferation through PEDF peptide stimulation at the wound site, suggesting that this is one of the mechanisms through which PEDF participates in wound healing. This idea opens up another frontier in promoting the wound healing process and may provide a therapeutic option for external wounds.

In addition to epithelium enhancement at the wound site, increased GT formation was observed under treatment with PEDF fragments. The mechanism of the accumulation of fibroblasts and extracellular matrix in GT remains to be determined. One of the postulated mechanisms may be associated with the fibroblast chemotactic function of PEDF (28); however, further evidence is required to determine the precise mechanism of action.

\section{Acknowledgements}

The authors would like to thank Dr Tim J Harrison for kindly proofreading this manuscript. Furthermore, the authors thank Chu-Ping Ho and Chin-Min Wang (Departments of Medical Research, Mackay Memorial Hospital, Taipei 10449) for assistance with animal experiments. The present study was supported by grants from the National Science Council, Taiwan (grant no. NSC-101-2314-B-195-006-MY3) and Mackay Memorial Hospital (MMH-E-101-006).

\section{References}

1. Wysocki AB: Wound fluids and the pathogenesis of chronic wounds. J Wound Ostomy Continence Nurs 23: 283-290, 1996.

2. Stojadinovic O, Ito $\mathrm{M}$ and Tomic-Canic M: Hair cycling and wound healing: To pluck or not to pluck? J Invest Dermatol 131: 292-294, 2011.

3. Werner S, Krieg T and Smola H: Keratinocyte-fibroblast interactions in wound healing. J Invest Dermatol 127: 998-1008, 2007.

4. Lau K, Paus R, Tiede S, Day P and Bayat A: Exploring the role of stem cells in cutaneous wound healing. Exp Dermatol 18: 921-933, 2009.

5. Szpaderska AM, Egozi EI, Gamelli RL and DiPietro LA: The effect of thrombocytopenia on dermal wound healing. J Invest Dermatol 120: 1130-1137, 2003.

6. Werner S and Grose R: Regulation of wound healing by growth factors and cytokines. Physiol Rev 83: 835-870, 2003.

7. Smola H, Thiekötter G and Fusenig NE: Mutual induction of growth factor gene expression by epidermal-dermal cell interaction. J Cell Biol 122: 417-429, 1993.

8. Stewart KJ: A quantitative ultrastructural study of collagen fibrils in human skin normal scars and hypertrophic scars. Clin Anat 8: 334-338, 1995.

9. Ito $\mathrm{M}$ and Cotsarelis $\mathrm{G}$ : Is the hair follicle necessary for normal wound healing? J Invest Dermatol 128: 1059-1061, 2008.

10. Ito M, Liu Y, Yang Z, Nguyen J, Liang F, Morris RJ and Cotsarelis G: Stem cells in the hair follicle bulge contribute to wound repair but not to homeostasis of the epidermis. Nat Med 11: 1351-1354, 2005.

11. Persson U, Willis M, Odegaard K and Apelqvist $\mathrm{J}$ : The cost-effectiveness of treating diabetic lower extremity ulcers with becaplermin (Regranex): A core model with an application using Swedish cost data. Value Health 3 (Suppl 1): S39-S46, 2000.

12. Steed DL: Clinical evaluation of recombinant human platelet-derived growth factor for the treatment of lower extremity ulcers. Plast Reconstr Surg 117 (7 Suppl): 143S-151S, 2006.

13. Chen L and DiPietro LA: Production and function of pigment epithelium-derived factor in isolated skin keratinocytes. Exp Dermatol 23: 436-438, 2014.

14. Ho TC, Chen SL, Wu JY, Ho MY, Chen LJ, Hsieh JW, Cheng HC and Tsao YP: PEDF promotes self-renewal of limbal stem cell and accelerates corneal epithelial wound healing. Stem Cells 31: 1775-1784, 2013. 
15. Ho TC, Chiang YP, Chuang CK, Chen SL, Hsieh JW, Lan YW and Tsao YP: PEDF-derived peptide promotes skeletal muscle regeneration through its mitogenic effect on muscle progenitor cells. Am J Physiol Cell Physiol 309: C159-C168, 2015.

16. Petersen SV, Valnickova Z and Enghild JJ: Pigmentepithelium-derived factor (PEDF) occurs at a physiologically relevant concentration in human blood: Purification and characterization. Biochem J 374: 199-206, 2003.

17. Blazejewska EA, Schlötzer-Schrehardt U, Zenkel M, Bachmann B, Chankiewitz E, Jacobi C and Kruse FE: Corneal limbal microenvironment can induce trans differentiation of hair follicle stem cells into corneal epithelial-like cells. Stem Cells 27: 642-652, 2009.

18. Snippert HJ, Haegebarth A, Kasper M, Jaks V, van Es JH, Barker N, van de Wetering $M$, van den Born $M$, Begthel $H$, Vries RG, et al: Lgr6 marks stem cells in the hair follicle that generate all cell lineages of the skin. Science 327: 1385-1389, 2010.

19. Filleur S, Volz K, Nelius T, Mirochnik Y, Huang H, Zaichuk TA, Aymerich MS, Becerra SP, Yap R, Veliceasa D, et al: Two functional epitopes of pigment epithelial-derived factor block angiogenesis and induce differentiation in prostate cancer. Cancer Res 65: 5144-5152, 2005.

20. Matsui T, Nishino Y, Maeda S and Yamagishi S: PEDF-derived peptide inhibits corneal angiogenesis by suppressing VEGF expression. Microvasc Res 84: 105-108, 2012.

21. Cai J, Jiang WG, Grant MB and Boulton M: Pigment epithelium-derived factor inhibits angiogenesis via regulated intracellular proteolysis of vascular endothelial growth factor receptor 1. J Biol Chem 281: 3604-3613, 2006.
22. Qi W, Yang C, Dai Z, Che D, Feng J, Mao Y, Cheng R, Wang Z, He X, Zhou T, et al: High levels of pigment epithelium-derived factor in diabetes impair wound healing through suppression of Wnt signaling. Diabetes 64: 1407-1419, 2015.

23. De Marzo A, Aruta C and Marigo V: PEDF promotes retinal neurosphere formation and expansion in vitro. Adv Exp Med Biol 664: 621-630, 2010.

24. Ramírez-Castillejo C, Sánchez-Sánchez F, Andreu-Agulló C, Ferrón SR, Aroca-Aguilar JD, Sánchez P, Mira H, Escribano J and Fariñas I: Pigment epithelium-derived factor is a niche signal for neural stem cell renewal. Nat Neurosci 9: 331-339, 2006.

25. Boxman ILA, Ruwhof C, Boerman OC, Löwik CWGM and Ponec M: Role of fibroblasts in the regulation of proinflammatory interleukin IL-1, IL-6 and IL-8 levels induced by keratinocyte-derived IL-1. Arch Dermatol Res 288: 391-398, 1996.

26. Waelti ER, Inaebnit SP, Rast HP, Hunziker T, Limat A Braathen LR and Wiesmann U: Co-culture of human keratinocytes on post-mitotic human dermal fibroblast feeder cells: Production of large amounts of interleukin 6. J Invest Dermatol 98: 805-808, 1992.

27. Florin L, Maas-Szabowski N, Werner S, Szabowski A and Angel P: Increased keratinocyte proliferation by JUN-dependent expression of PTN and SDF-1 in fibroblasts. J Cell Sci 118: 1981-1989, 2005.

28. Sarojini H, Estrada R, Lu H, Dekova S, Lee MJ, Gray RD and Wang E: PEDF from mouse mesenchymal stem cell secretome attracts fibroblasts. J Cell Biochem 104: 1793-1802, 2008. 